\title{
Multi-Domain Pseudospectral Time-Domain Simulations of Scattering by Objects Buried in Lossy Media
}

\author{
Guo-Xin Fan and Qing Huo Liu \\ Department of Electrical and Computer Engineering \\ Duke University \\ Durham, NC 27514 \\ Jan S. Hesthaven \\ Division of Applied Mathematics \\ Brown University \\ Providence, RI 02912
}

\begin{abstract}
A multidomain pseudospectral time-domain (PSTD) method with a suitable well-posed PML is introduced as an accurate and flexible tool for the modeling of electromagnetic scattering by 2-D objects buried in an inhomogeneous lossy medium. Compared with the previous Fourier PSTD method, this approach allows for an accurate treatment of curved geometries as confirmed by numerical results showing an excellent agreement with analytical solutions for scattering by perfectly conducting as well as permeable dielectric cylinders. The algorithm has been applied to model various ground penetrating radar (GPR) applications involving interfaces of an arbitrary shape and in a lossy half space with an undulating surface. The numerical results demonstrate the effectiveness of the multidomain PSTD algorithm as an accurate, flexible and robust approach for the time-domain solution of Maxwell's equations.
\end{abstract}

Index Terms - Finite-difference time-domain (FDTD) method, pseudospectral timedomain (PSTD) method, domain decomposition, perfectly matched layer (PML), ground penetrating radar (GPR), rough surface. 


\section{Introduction}

The finite-difference time-domain (FDTD) method [1] has been widely used to simulate propagation, scattering, and radiation of electromagnetic waves although it is well known that the conventional FDTD method has a second-order spatial and temporal accuracy. As has been verified through numerous examples this requires that a sampling density of 10-20 cells per minimum wavelength is used to ensure that the FDTD method produces acceptable results.

In recent years, higher-order and spectral methods $[2,3]$ have received increasing attention as an efficient and robust computational technique with numerous applications in computational fluid mechanics [3] and, more recently, in computational electromagnetics . For the time-domain solution of Maxwell's equations, two different pseudospectral techniques have been introduced. The first approach, the Fourier pseudospectral time-domain (PSTD) algorithm [4, 5, 6, 7], combines the fast Fourier transform algorithm with the perfectly matched layer (PML) absorbing boundary condition to enable fast modeling of scattering and penetration problems in simple geometries. The second approach is centered around a pseudospectral multi-domain method $[8,9,10,11]$ to allow a flexible and accurate representation of the geometry and potential for highly parallel implementations. The central idea of the PSTD methods is to use polynomials to express the unknown solution and its spatial derivatives to achieve a much more accurate representation of the solution as compared to the classical FDTD approach. While the Fourier PSTD method is simple to implement and requires only two points per minimum wavelength in homogeneous and smoothly varying inhomogeneous regions, its accuracy is lower when applied to more complex problems involving curved boundaries, perfectly conducting bodies, and discontinuous material distributions. The multidomain PSTD overcomes these restrictions and can deal with complex objects with a great flexibility, but with a slightly increased computational burden as it requires $\pi$ cells per minimum wavelength. Both methods have been shown to be more efficient than the FDTD method for many applications.

Previously, the Fourier PSTD method has been applied to model ground penetrating radar (GPR) detection of buried dielectric objects. Although this approach has significant computational advantages over the FDTD method, a number of aspects of this approach need further improvement, i.e., the geometric modeling of curved objects, the modeling of perfect conductors, and the correct treatment of material interfaces, in which case the globaldomain approach of the Fourier PSTD method looses accuracy due the loss of smoothness of the solutions. In such cases, the multidomain PSTD approach is a suitable alternative. In the multidomain approach, the computational domain is divided into several subdomains conforming to the problem geometry. A polynomial approximation is then used in each subdomain as in the global approach, and the solution across subdomains are combined using patching conditions derived in accordance with the physics of the problem to recover the global solution. 
The multidomain PSTD method retains the accuracy of the global PSTD method and has several important advantages over its global counterpart: (a) geometric flexibility and the ability to model material interfaces and metallic boundaries correctly; (b) reduction in the computational cost when increasing the number of subdomain; and (c) potential for efficient parallel implementation.

In this work, we apply the multidomain PSTD algorithm to lossy media, and incorporate a well-posed PML for lossy media for the truncation of the computational domain. Unlike most previous applications of the multidomain PSTD approach, using a sinusoidal field excitation, we demonstrate the flexibility and accuracy of the scheme when applied to wideband GPR scenarios. For this particular application we found it necessary to develop a localized source representation suitable for the multidomain PSTD method.

What remains of the paper is organized as follows. In Sec. II we briefly recall the physical problem and the setup of a typical GPR scenario. This sets the stage for Sec. III where we discuss the numerical scheme in detail, paying attention to the body-conforming discretization and the high-order representation of the solution as well as the local patching scheme, the time-advancement scheme, and the PML for lossy media. The efficacy of the complete framework is addressed in Sec. IV where we present validation examples as well as demonstrate the accuracy, flexibility, and robustness of the scheme on more realistic scenarios of relevance to GPR analysis and development. Section V contains a few concluding remarks and guidelines for future work within the present context.

\section{Physical Problem}

We consider an isotropic, conductive, inhomogeneous medium with electric permittivity $\epsilon$, magnetic permeability $\mu$, and conductivity $\sigma$. A typical GPR detection scenario is shown in Figure 1, illustrating an undulating ground surface and complex objects buried in the earth.

For the two-dimensional $\mathrm{TM}_{z}$ (transverse magnetic to $z$ ) polarization, Maxwell's equations take the form

$$
\begin{aligned}
\frac{\partial H_{x}}{\partial t} & =-\frac{1}{\mu} \frac{\partial E_{z}}{\partial y} \\
\frac{\partial H_{y}}{\partial t} & =\frac{1}{\mu} \frac{\partial E_{z}}{\partial x} \\
\frac{\partial E_{z}}{\partial t} & =\frac{1}{\epsilon}\left(\frac{\partial H_{y}}{\partial x}-\frac{\partial H_{x}}{\partial y}\right)-\frac{\sigma}{\epsilon} E_{z}
\end{aligned}
$$

where $\epsilon, \mu$, and $\sigma$ are only functions of $(x, y)$. 


\section{Numerical Scheme}

The strategy for solving Maxwell's equations using the multidomain Chebyshev PSTD method is as follows. (i) The computational domain is divided into non-overlapping subdomains, conforming with the problem geometry. For a general inhomogeneous medium each block contains only smoothly varying materials. Dividing one block of homogeneous material into several subdomains is based on computational efficiency consideration. In general, interfaces between subdomains may have the same or different materials on both sides. (ii) At each time step, the fields are first calculated independently within each subdomain, and then (iii) patching conditions are applied to reconcile the fields from adjacent subdomains to complete field calculation at one time step. (iv) An explicit Runge-Kutta method is used to advance the solution to the next time step. In the following we shall discuss these individual components in more detail.

\section{III.1 Maxwell's Equations on Curvilinear Form}

The traditional global-domain PSTD method requires coordinate directions parallel to the boundary in the domain to avoid staircasing errors as well as a high degree of smoothness of the solutions to allow a high order accuracy. Here, we will use a multidomain formulation to enable the accurate modeling of problems with curved subdomains and the representation of piecewise smooth solutions.

To facilitate this we use a coordinate transformation to map a subdomain with curved boundaries in $(x, y)$ coordinates into a regular domain in $(\xi, \eta)$ coordinates as shown in Fig. 2 . Introducing a general curvilinear coordinates with the transform relation,

$$
\xi=\xi(x, y), \quad \eta=\eta(x, y)
$$

Maxwell's equations, Eqs. (1)-(3), can be written in the matrix form

$$
\frac{\partial \boldsymbol{q}}{\partial t}+A \frac{\partial \boldsymbol{q}}{\partial \xi}+B \frac{\partial \boldsymbol{q}}{\partial \eta}+C \boldsymbol{q}=0
$$

where $\boldsymbol{q}=\left(H_{x}, H_{y}, E_{z}\right)^{T}$,

$$
A=\left(\begin{array}{ccc}
0 & 0 & \xi_{y} / \mu \\
0 & 0 & -\xi_{x} / \mu \\
\xi_{y} / \epsilon & \xi_{x} / \epsilon & 0
\end{array}\right), \quad B=\left(\begin{array}{ccc}
0 & 0 & \eta_{y} / \mu \\
0 & 0 & -\eta_{x} / \mu \\
\eta_{y} / \epsilon & \eta_{x} / \epsilon & 0
\end{array}\right), \quad C=\left(\begin{array}{ccc}
0 & 0 & 0 \\
0 & 0 & 0 \\
0 & 0 & \sigma / \epsilon
\end{array}\right)
$$

and $\xi_{x}=\partial \xi / \partial x, \xi_{y}=\partial \xi / \partial y$ and so on. With this transformation, we can then solve Eq. $(5)$ in the $(\xi, \eta)$ coordinates for a general subdomain. 


\section{III.2 Chebyshev Spectral Collocation Procedure}

For 2-D problems, we assume that the computational domain can be broken into a set of non-overlapping curvilinear quadrilaterals, conforming to the geometry and material distribution. Using transfinite blending functions [13], each quadrilateral in $(x, y)$ coordinates can be mapped onto the unit square, i.e., $[-1,1] \times[-1,1]$, in $(\xi, \eta)$ coordinates and we can thus concern ourselves with the formulation of the scheme on this unit element.

Our aim is to accurately evaluate the spatial derivatives of functions, $q(\xi, \eta)$, defined on the unit element. For this we shall use a tensor-product Chebyshev method based on the Chebyshev-Gauss-Lobatto collocation points

$$
\begin{aligned}
\xi_{i} & =-\cos (i \pi / N), & i & =0,1, \ldots N, \\
\eta_{j} & =-\cos (j \pi / M), & j & =0,1, \ldots M .
\end{aligned}
$$

In this setting we assume that $q(\xi, \eta)$ is well represented by a tensor-product ChebyshevLagrange polynomial of the form

$$
q(\xi, \eta)=\sum_{i=0}^{N} \sum_{j=0}^{M} q\left(\xi_{i}, \eta_{j}\right) g_{i}(\xi) g_{j}(\eta)
$$

where the Lagrange interpolation polynomials are given as

$$
g_{i}(\alpha)=\frac{\left(1-\alpha^{2}\right) T_{N}^{\prime}(\alpha)(-1)^{i+1}}{c_{i} N^{2}\left(\alpha-\alpha_{i}\right)} .
$$

Here $\alpha=(\xi, \eta), c_{0}=c_{N}=2$ and $c_{i}=1$ otherwise, and the $N$ th-order Chebyshev polynomial is defined as $T_{N}(x)=\cos \left(N \cos ^{-1} x\right)$.

Under this assumption partial derivatives of $q(\xi, \eta)$ at the grid points are obtained as

$$
\begin{aligned}
& \frac{\partial q\left(\xi_{k}, \eta_{j}\right)}{\partial \xi}=\sum_{k=0}^{N} D_{i k}^{(\xi)} q\left(\xi_{i}, \eta_{j}\right), \\
& \frac{\partial q\left(\xi_{i}, \eta_{j}\right)}{\partial \eta}=\sum_{k=0}^{M} D_{j k}^{(\eta)} q\left(\xi_{i}, \eta_{k}\right),
\end{aligned}
$$

where the elements of the differentiation matrix, $D_{i k}^{(\alpha)}$, are given as

$$
D_{i k}^{(\alpha)}=g_{k}^{\prime}\left(\alpha_{i}\right), \quad \alpha=(\xi, \eta),
$$


and the explicit expressions are given as [3]

$$
D_{i k}^{(\alpha)}=\left\{\begin{array}{ll}
\frac{c_{i}}{c_{k}} \frac{(-1)^{i+k}}{\alpha_{i}-\alpha_{k}} & i \neq k \\
\frac{-\alpha_{k}}{2\left(1-\alpha_{k}^{2}\right)} & 1 \leq i=k \leq N-1 \\
\frac{2 N^{2}+1}{6} & i=k=0 \\
-\frac{2 N^{2}+1}{6} & i=k=N .
\end{array} .\right.
$$

Hence, the evaluation of spatial derivatives are accomplished by matrix-vector multiplies, similar to classical finite difference methods, yet with the potential for very high accuracy for smooth solutions using only very few grid points.

\section{III.3 Characteristic Variables}

So far we have concerned ourselves with the evaluation of the general curvilinear equations within a single element. However, to recover the global solution one needs to pass information between the individual subdomains in a way consistent with the nature of the problem.

For this purpose we shall exploit the characteristic variables associated with the hyperbolic system of equations, Eq. (5). We can diagonalize $A$ as [9]

$$
A=S \Lambda S^{-1}
$$

where

$$
S=\left(\begin{array}{ccc}
-\hat{\xi}_{y} & \hat{\xi}_{x} & -\hat{\xi}_{y} \\
\hat{\xi}_{x} & \hat{\xi}_{y} & \hat{\xi}_{x} \\
\sqrt{\frac{\mu}{\epsilon}} & 0 & -\sqrt{\frac{\mu}{\epsilon}}
\end{array}\right), \quad S^{-1}=\frac{1}{2}\left(\begin{array}{ccc}
-\hat{\xi}_{y} & \hat{\xi}_{x} & \sqrt{\frac{\epsilon}{\mu}} \\
2 \hat{\xi}_{x} & 2 \hat{\xi}_{y} & 0 \\
-\hat{\xi}_{y} & \hat{\xi}_{x} & -\sqrt{\frac{\epsilon}{\mu}}
\end{array}\right)
$$

and

$$
\Lambda=\left(\begin{array}{ccc}
-\sqrt{\frac{\xi_{x}^{2}+\xi_{y}^{2}}{\mu \epsilon}} & 0 & 0 \\
0 & 0 & 0 \\
0 & 0 & \sqrt{\frac{\xi_{x}^{2}+\xi_{y}^{2}}{\mu \epsilon}}
\end{array}\right)
$$


The resulting characteristic vector can be written as

$$
\boldsymbol{R}=S^{-1} \boldsymbol{q}=\left(\begin{array}{c}
R_{-} \\
R_{0} \\
R_{+}
\end{array}\right)=\frac{1}{2}\left(\begin{array}{c}
-\hat{\xi}_{y} H_{x}+\hat{\xi}_{x} H_{y}+\sqrt{\frac{\epsilon}{\mu}} E_{z} \\
2 \hat{\xi}_{x} H_{x}+2 \hat{\xi}_{y} H_{y} \\
-\hat{\xi}_{y} H_{x}+\hat{\xi}_{x} H_{y}-\sqrt{\frac{\epsilon}{\mu}} E_{z}
\end{array}\right) .
$$

The above characteristic variables reflect the amplitudes of the characteristic waves propagating along $-\xi$, non-propagating, and along $+\xi$, respectively, while the eigenvalues, i.e. the diagonal entries of $\Lambda$, correspond to their propagation speeds. The characteristic variables of matrix $B$ have a form similar to those of matrix $A$.

In a general scenario there are two distinct types of interfaces: one that separates two subdomains with different materials and one that separates two subdomains with the same material. The matching conditions for these two types of interfaces are different.

First consider an interface separating two subdomains of the same material. Suppose the $\xi$ axis points from subdomain 1 to subdomain 2 . In this case, we match the characteristic waves at the interface as

$$
\begin{aligned}
R_{-}^{(1)} & \leftarrow R_{-}^{(2)} \\
R_{+}^{(2)} & \leftarrow R_{+}^{(1)} \\
R_{0}^{(1,2)} & =\frac{1}{2}\left(R_{0}^{(1)}+R_{0}^{(2)}\right)
\end{aligned}
$$

where the superscript denotes the two different subdomains. Essentially, these conditions reflect that the incoming characteristic wave is determined by the out-going characteristic wave from the adjacent subdomain, the non-propagating characteristic wave remains continuous and the out-going characteristic wave is left unaltered to enter the next domain.

For an interface separating two subdomains of different materials, physical boundary conditions have to be applied. For dielectric interfaces, this implies continuity of the tangential electric and magnetic field components while for PEC interfaces we enforce a vanishing tangential electric and normal magnetic field components.

\section{III.4 Time Stepping}

To advance (5) in time we use a 4th order 5 stage low-storage version of the classical Runge-Kutta method. Expressing (5) as

$$
\frac{\partial \boldsymbol{q}}{\partial t}=\boldsymbol{f}(t, \boldsymbol{q})
$$

where

$$
\boldsymbol{f}=-\left(A \frac{\partial \boldsymbol{q}}{\partial \xi}+B \frac{\partial \boldsymbol{q}}{\partial \eta}+C \boldsymbol{q}\right)
$$


Denoting $\boldsymbol{q}\left(t_{n+1}\right)$ as $\boldsymbol{q}_{n+1}$ where $t_{n}=n \Delta t$ and $\Delta t$ is the time step size, the low-storage form of the Runge-Kutta method is given as

$$
\begin{aligned}
& \boldsymbol{q}_{0}=\boldsymbol{q}_{n} \\
\forall j \in[1,5]: \quad & \left\{\begin{array}{l}
\boldsymbol{k}_{j}=a_{j} \boldsymbol{k}_{j-1}+\Delta t \boldsymbol{f}\left(\left(n+c_{j}\right) \Delta t, \boldsymbol{q}_{j}\right) \\
\boldsymbol{q}_{j}=\boldsymbol{q}_{j-1}+b_{j} \boldsymbol{k}_{j} \\
\boldsymbol{q}_{n+1}=\boldsymbol{q}_{5}
\end{array}\right.
\end{aligned}
$$

where the constants $a_{j}, b_{j}$ and $c_{j}$ are determined to yield the desired order, $s-1$, of the scheme. For the scheme to be self-starting we require that $a_{1}=0$. Note that we need only two storage levels containing, $\boldsymbol{k}_{j}$ and $\boldsymbol{q}_{j}$, to advance the solution. The actual values of $a_{j}$, $b_{j}$, and $c_{j}$ can be found in [12]. The choice of the time step was discussed in [9].

\section{III.5 A Well-Posed PML for Conductive Media}

A key issue in the time-domain solution is the introduction of an absorbing boundary condition to truncate the unbounded medium to enable the solution in a finite computational domain without introducing noticeable reflections from the computational edge. One recent significant breakthrough is the perfectly matched layer proposed by Berenger [14]. The original PML formulation by Berenger, as well as those later developed by Chew and Weedon [15] and extended to lossy media by Liu [16], uses the split-field formulation. Such splitfield schemes have later been shown to be only weakly well-posed [17]. Thus there has been

significant investigations on well-posed PML formulations (e.g., [18, 19, 20, 21, 22]). Most of these well-posed PML formulations have been proposed for lossless media. Here we use a systematic method [23] to derive a well-posed PML for a lossy medium.

Following $[15,16]$, we introduce complex coordinate-stretching variables as

$$
\partial x \Rightarrow\left[1+\frac{i \omega_{x}(x)}{\omega}\right] \partial x \quad \partial y \Rightarrow\left[1+\frac{i \omega_{y}(y)}{\omega}\right] \partial y .
$$

Defining new field variables for the PML region [21]

$$
\widetilde{H}_{x}=H_{x}+\omega_{x} Q_{x}, \quad \widetilde{H}_{y}=H_{y}+\omega_{y} Q_{y}
$$

we can rewrite Eqs. (1)-(3) for the PML as

$$
\begin{aligned}
& \frac{\partial \widetilde{H}_{x}}{\partial t}=-\frac{1}{\mu} \frac{\partial E_{z}}{\partial y}+\left(\omega_{x}-\omega_{y}\right)\left(\widetilde{H}_{x}-\omega_{x} Q_{x}\right) \\
& \frac{\partial \widetilde{H}_{y}}{\partial t}=\frac{1}{\mu} \frac{\partial E_{z}}{\partial x}+\left(\omega_{y}-\omega_{x}\right)\left(\widetilde{H}_{y}-\omega_{y} Q_{y}\right)
\end{aligned}
$$




$$
\begin{aligned}
\frac{\partial E_{z}}{\partial t}= & \frac{1}{\epsilon}\left(\frac{\partial \widetilde{H}_{y}}{\partial x}-\frac{\partial \widetilde{H}_{x}}{\partial y}\right)-\frac{\sigma}{\epsilon} E_{z}-\left(\omega_{x}+\omega_{y}\right) E_{z} \\
& -\left[\frac{\sigma}{\epsilon}\left(\omega_{x}+\omega_{y}\right)+\omega_{x} \omega_{y}\right] P_{z}-\frac{\sigma}{\epsilon} \omega_{x} \omega_{y} K_{z}
\end{aligned}
$$

where

$$
\begin{aligned}
& \frac{\partial Q_{x}}{\partial t}=\widetilde{H}_{x}-\omega_{x} Q_{x} \\
& \frac{\partial Q_{y}}{\partial t}=\widetilde{H}_{y}-\omega_{y} Q_{y} \\
& \frac{\partial P_{z}}{\partial t}=E_{z} \\
& \frac{\partial K_{z}}{\partial t}=P_{z} .
\end{aligned}
$$

Note that this non-split PML is well-posed for conductive media because Eq. (23) remains the same symmetric hyperbolic system as the original Maxwell's equations plus some lower order terms that do not affect the well-posedness [24, 17]. When $\sigma=0$, the PML equations reduce to ones known for lossless media [19, 21, 20]. When $\omega_{x}=\omega_{y}=0$, the PML equations reduce to Maxwell's equations for a regular medium. In addition, Eqs. (25)-(28) are ordinary differential equations without spatial derivatives, ensuring a simple and efficient implementation.

The patching condition at an interface between a PML subdomain and a regular medium with the same physical properties $(\epsilon, \mu, \sigma)$ is the continuity of tangential $\widetilde{\mathbf{E}}$ and tangential $\widetilde{\mathbf{H}}$. At the outer boundary of the PML subdomain, the incoming characteristic variable is set zero so that waves are purely outgoing.

\section{III.6 Source excitation treatments}

Two types of excitation sources are considered in this work: uniform plane waves and localized line sources. For plane wave excitation, the total-field/scattered-field formulation is used. In this approach, the scattered field is calculated in the PML region while the total field is used in the interior region.

For the line source excitation, special care must be taken because a line source represents a delta function, which cannot be accurately represented by Chebyshev polynomials. To deal with this problem one may use a spatially smoothed source, i.e., a set of line sources with a smooth amplitude distribution, to approximate the line source $[4,5]$. In this approach, the total field formulation is used in the whole computational domain. This smoothed source 
usually gives an acceptable accuracy when source remains away from a subdomain interface. This approach is suitable to the applications where a uniform grid or a fixed source is used.

However, for GPR applications, the instrument, and thus the source, moves along the surface of the ground. When the source moves close to an interface between subdomains, the above smoothed source treatment will give rise to inaccurate result since the grid is nonuniform and the available zone for source smoothing is reduced. To overcome this problem, we use a scattered-field/total-field formulation. In the subdomain where the source is located, the scattered field is calculated, while the total field is calculated in the remaining subdomains. To match the boundary condition on the boundary of the source subdomain, a precalculated primary field due to a single line source in a homogeneous medium is used for construction of the total field on the boundaries of the source subdomain. The increase in computational cost is minimal as this primary field is only required on the boundary of one subdomain.

In terms of the temporal excitation function, there are two kinds of GPR systems, i.e., systems with short-pulse excitation and those with step frequencies (sinusoidal excitation). The following results will be presented for the short-pulse excitation where the BlackmanHarris window function $[4,16]$ is used as the time functions for both the plane wave excitation and the line source excitation.

\section{Numerical Results}

The computational domain of rectangular shape is divided into non-overlapping quadrilaterals. The outer layer of quadrilaterals is used as PML subdomains in which the PML lossy media is applied using a second order polynomial absorption profile. Each quadrilateral is meshed with a grid where the grid points are located at the Chebyshev-Gauss-Lobatto collocation points. In all examples, a grid with $16 \times 16$ points are used for each subdomain. The time function of the plane wave source and the line sources is chosen as the Blackman-Harris window function.

As our first test example, we consider the plane wave scattering from a PEC cylinder in free space. Fig. 3(a) illustrates the decomposition and Fig. 3(b) shows the grid used in the calculation. A plane wave is incident on the cylinder along $x$ axis. The central frequency of the plane wave is $100 \mathrm{MHz}$, corresponding to $k a=4 \pi / 5$. In the calculation, $\Delta t=40 \mathrm{ps,}$ and the receiver is located at $x=y=-2.1036 \mathrm{~m}$. Fig. 3(c) compares the PSTD numerical results with the analytical solution, showing excellent agreement.

The second example is a dielectric circular cylinder with $\epsilon_{r}=4$ and $\mu_{r}=1$. The geometry and source are similar to those in the first example as illustrated in Figs. 4(a,b). The time step size is $\Delta t=21.5$ ps. Figure $4(\mathrm{c})$ shows the comparison of the numerical and analytical solution. Again we find excellent agreement.

The third test example considers the field due to a line source (central frequency 100 
$\mathrm{MHz}$ ) at the center of an air filled cylinder in a homogeneous lossy medium. In this example, the grid used is the same as in the first examples and the parameters of the outer material are $\epsilon_{r}=4, \mu_{r}=1$, and $\sigma=0.002 \mathrm{~S} / \mathrm{m}$. To show the effect of the permeability, we also consider the case in which the relative permeability of the cylinder is changed from $\mu_{r}=1$ to $\mu_{r}=4$. The time step size is $\Delta t=21.5 \mathrm{ps}$, and the receiver location is the same as in the above examples. Figure 5 shows the excellent agreement between the numerical result and the analytical solution for both cases. For this problem, the minimum wavelength at $f=3 f_{c}=150 \mathrm{MHz}$ is $\lambda_{\min }=0.5 \mathrm{~m}$, while the average cell size is $\bar{\Delta} x=0.125 \mathrm{~m}$, or $1 / 4$ of the minimum wavelength.

In the above examples, curved interfaces have been accurately modeled by the multidomain PSTD through coordinate transformation to curvilinear coordinates. This procedure makes the numerical results highly accurate without the staircasing error, even with the average sampling rate being as low as 4 cells per wavelength.

In the following, we consider the applications of the multidomain PSTD to GPR detection of buried objects. In these examples, the electric parameters of the earth are $\epsilon_{r}=4, \mu_{r}=1$ and $\sigma=0.002 \mathrm{~S} / \mathrm{m}$. The central frequency of the line source is $300 \mathrm{MHz}$. The scattered field is defined as the total field in the presence of buried objects subtracted by the field in the absence of buried objects.

As a first example we consider three cylinders of different shape and electric parameters being buried in a lossy half space. Figs. 6(a) and 6(b) show the geometry and the grid. A line source and a receiver at a fixed distance are moving along the interface between air and earth. The scattered field from the buried objects is plotted in grad-level form as shown in Fig. 6(c).

The second GPR example is for two circular cylinders, one PEC and the other dielectric $\left(\epsilon_{r}=2, \sigma=0\right)$, buried in a lossy half-space. Figs. 7(a) and 7(b) show the geometry and the distribution of the scattered field, respectively.

To demonstrate the efficiency and flexibility of the multidomain PSTD algorithm, we consider the effects of the rough surface on the scattering by buried objects. A square PEC cylinder is buried in a lossy medium, but the air/soil interface is undulated as shown in Fig. 8(a). The source and receiver are moving together along the surface at $y=0$. Fig. 8 (b) shows the total electric field distribution in the presence of the undulating surface. For comparison, we also show the field distribution when the rough surface is replaced by a plane surface in Fig. 8 (c). The effect of the undulating surface is strong as clearly seen by comparing the two figures. Nevertheless, in spite of the undulating surface, the scattered field clearly shows the presence of the buried object.

\section{Conclusions}

A multidomain pseudospectral time-domain (PSTD) scheme is applied to model electro- 
magnetic scattering by 2-D objects buried in lossy media. The formulation incorporates a well-posed PML for lossy media and utilizes a scattered-field/total-field formulation to enable the excitation of a localized moving source in GPR applications. The numerical results show an excellent agreement with analytical solutions for various canonical problems. The algorithm has been applied to model various GPR applications involving interfaces of arbitrary shape in a lossy half space and the numerical results demonstrate the efficiency and robustness of the multidomain PSTD algorithm as a body-conforming high-order accurate time-domain solver for Maxwell's equations.

\section{Acknowledgment}

The work of the first two authors were supported by the U.S. EPA through a PECASE grant CR-825-225-010, and by the NSF through a CAREER grant ECS-9702195. The third

author acknowledges partial support by AFOSR/DARPA under contract F49620-1-0426 and by the Alfred P. Sloan Foundation as a Sloan Research Fellow.

\section{REFERENCES}

1. K. S. Yee, "Numerical solution of initial boundary value problems involving Maxwell's equations in isotropic media," IEEE Trans. Antennas Propagat., vol. 14, pp. 302-307, May, 1966.

2. D. Gottlieb, and S. A. Orszag, Numerical Analysis of Spectral Methods, SIAM, Philadelphia, 1977.

3. C. Canuto, M. Y. Hussani, A. Quarteroni, and T. Zang, Spectral Methods in Fluid Dynamics, Springer-Verlag, New York, 1988.

4. Q. H. Liu, "The PSTD algorithm: A time-domain method requiring only two cells per wavelength" Microwave Opt. Technol. Lett., vol. 15, pp.158-165, 1997.

5. Q. H. Liu, "Large-scale simulations of electromagnetic and acoustic measurements using the pseudospectral time-domain (PSTD) algorithm" IEEE Trans. Geosci. Remote Sensing, vol. 37, no. 2, pp. 917-926, 1999.

6. Q. H. Liu, and G.-X. Fan, "A frequency-dependent PSTD algorithm for general dispersive media" IEEE Microwave Guided Wave Lett., vol. 9, pp. 51-53., 1999.

7. Q. H. Liu and G.-X. Fan, "Simulations of GPR in dispersive media using a frequencydependent PSTD algorithm" IEEE Trans. Geosci. Remote Sensing, vol. 37(5), 2317-2324, 1999.

8. B. Yang, D. Gottieb, and J. S. Hesthaven, "Spectral simulations of electromagnetic wave scattering" J. Comput. Phy., vol. 134, pp. 216-230, 1997.

9. J. S. Hesthaven, P. G. Dinesen, and J. P. Lynov, "Spectral collocation time-domain modeling of diffractive optical elements" J. Comput. Phy., vol. 155, pp. 287-306, 1999. 
10. B. Yang and J. S. Hesthaven, "A pseudospectral method for time-domain computation of electromagnetic scattering by bodies of revolution" IEEE Trans. Antennas Propagat., vol. 47, pp. 132-141, 1999.

11. B. Yang and J. S. Hesthaven, "Multidomain Pseudospectral Computation of Maxwell's Equations in 3-D General Curvilinear Coordinates" Appl. Numer. Math. vol. 33, pp. 281-289, 2000.

12. M. H. Carpenter and C. A. Kennedy, Fourth Order $2 N$-Storage Runge-Kutta Scheme, Technical Report, NASA-TM-109112, 1994.

13. W. J. Gordon and C. A. Hall, "Transfinite element methods: Blending function interpolation over arbitrary curved element domains," Numer. Math., vol. 21, pp. 109-129, 1973.

14. J. P. Berenger, "A Perfectly matched layer for the absorption of electromagnetic waves," $J$. Comput. Phys., vol. 114, pp. 185-200, 1994.

15. W. C. Chew and W. H. Weedon, "A 3D perfectly matched medium from modified Maxwell's equation with stretched coordinates," Microwave Opt. Tech. Lett., vol. 7, pp. 599-604, 1994.

16. Q. H. Liu, "An FDTD algorithm with perfectly matched layers for conductive media," $M i$ crowave Opt. Tech. Lett., vol. 14, pp. 134-137, 1997.

17. S. Abarbanel and D. Gottlieb, "A Mathematical Analysis of the PML Method," J. Comput. Phys., vol. 134, pp. 357-363.

18. S. Gedney, "An anisotropic perfectly matched layer - Absorbing medium for the truncation of FDTD lattices," IEEE Trans. Antennas Propagat., vol. 44, pp. 1630-1639, 1996.

19. R. W. Ziolkowski, "Time-Derivative Lorentz-material model based absorbing boundary conditions", IEEE Trans. Antennas Propagat., vol. 45, pp. 1530-1535, 1997.

20. S. Abarbanel and D. Gottlieb, "On the Construction and Analysis of Absorbing Layers in CEM", Appl. Numer. Math., vol. 27, pp. 331-340, 1998.

21. E. Turkel and A. Yefet, "Absorbing PML boundary layers for wave-like equations," Appl. Numer. Math., vol. 27, pp. 533-557, 1998.

22. D. C. Wittwer and R. W. Ziolkowski, "Maxwellian material-based absorbing boundary conditions for lossy media in 3-D," IEEE Trans. Antennas Propagat., vol. 48, pp. 200-213, 2000.

23. G.-X. Fan and Q. H. Liu, "A well-posed PML absorbing boundary condition for lossy media," Submitted to Microwave Opt. Tech. Lett.

24. B. Gustafsson, H. O. Kreiss, and J. Oliger, Time Dependent Problems and Difference Methods. New York: Wiley, 1995. 


\section{FIGURE CAPTIONS}

Fig. 1. A typical GPR measurement geometry and computational domain.

Fig. 2. Domain mapping from a curved quadrilateral to a unit square.

Fig. 3. Plane wave scattering from a PEC cylinder in free space. (a) Geometry and subdomains. (b) Grid. (c) Comparison the PSTD result with analytical solution.

Fig. 4. Plane wave scattering from a dielectric cylinder in free space. (a) Geometry and subdomains. (b) Grid. (c) Comparison the PSTD result with analytical solution.

Fig. 5. Field due to line source at the center of a cylinder with $\epsilon_{r}=1$, (a) $\mu_{r}=1$ and (b) $\mu_{r}=4$ in a lossy medium.

Fig. 6. Scattering by objects buried in a lossy half space. (a) Geometry. (b) Grid. (c) Scattered field distribution.

Fig. 7. Scattering from buried PEC and dielectric cylinders in a lossy half space. (a) Geometry. (b) Scattered field distribution.

Fig. 8. Scattering from a PEC square cylinder and an undulating ground surface. (a) Geometry. (b) Total field distribution in presence of a rough surface. (c) Total field distribution in presence of a plane surface. 


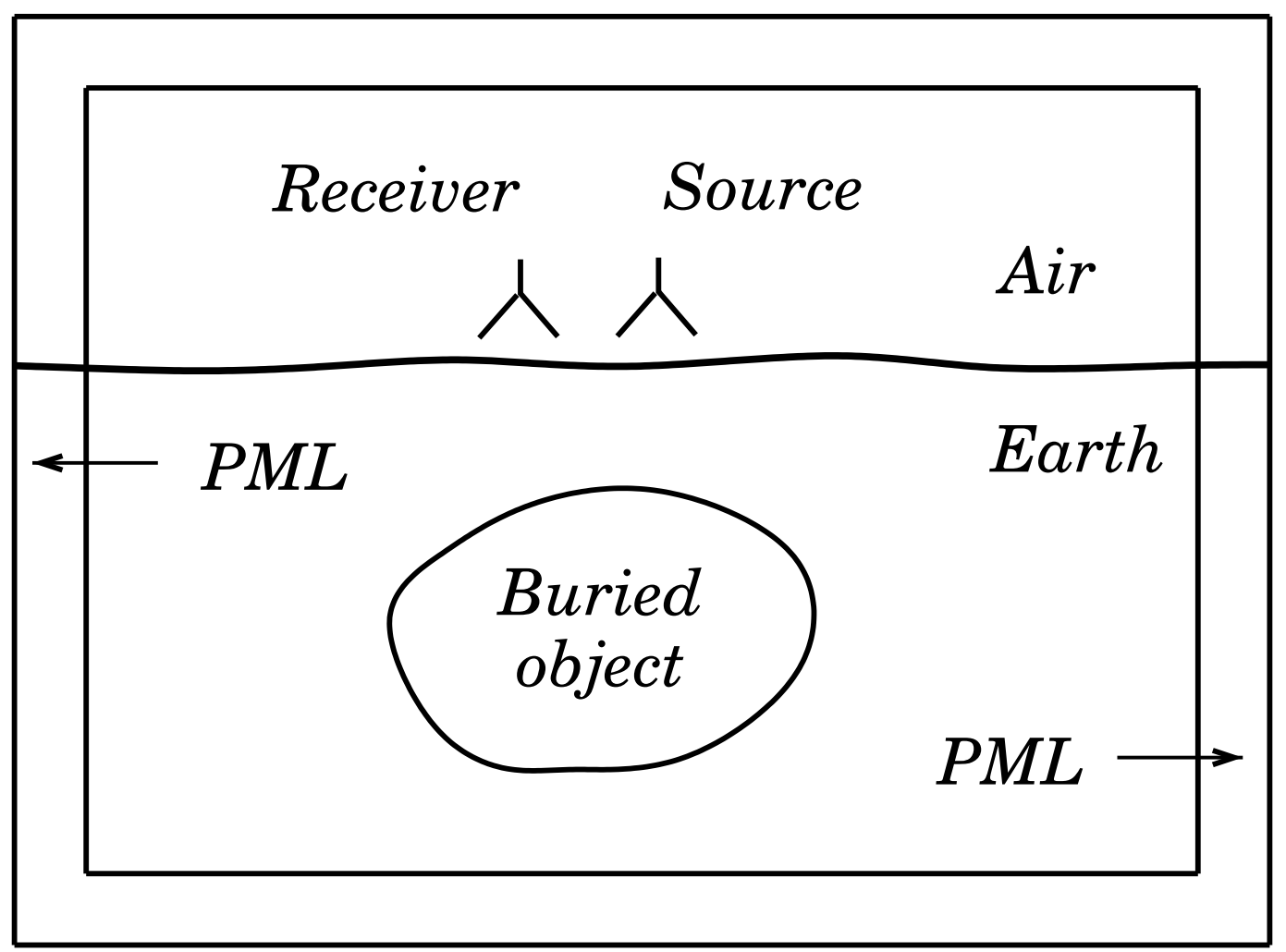

Fig. 1 

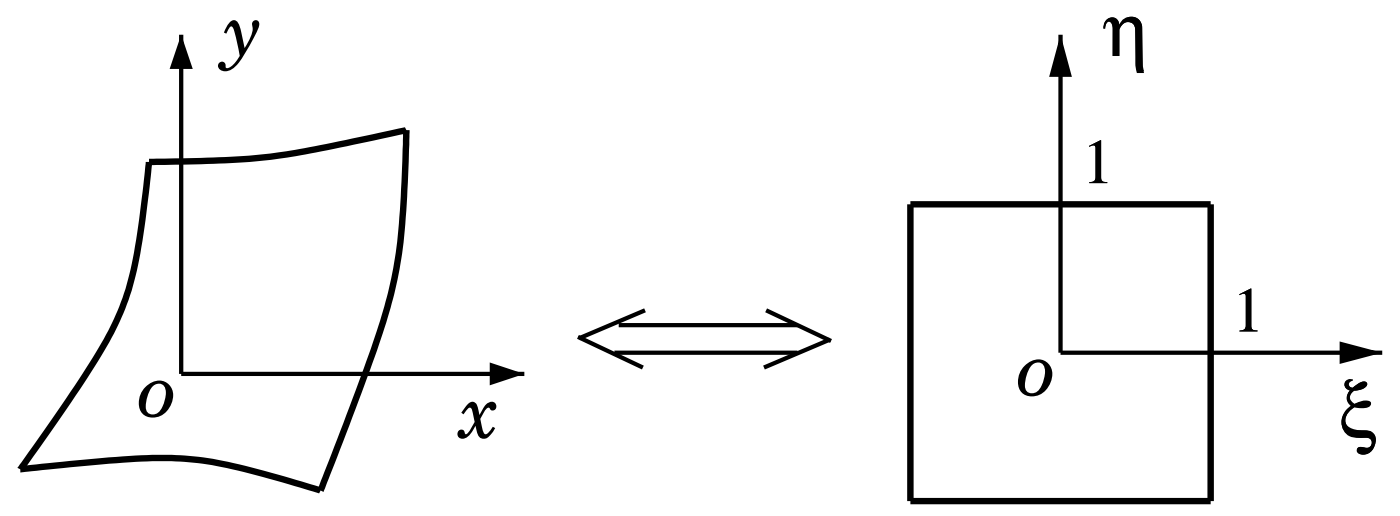

Fig. 2 


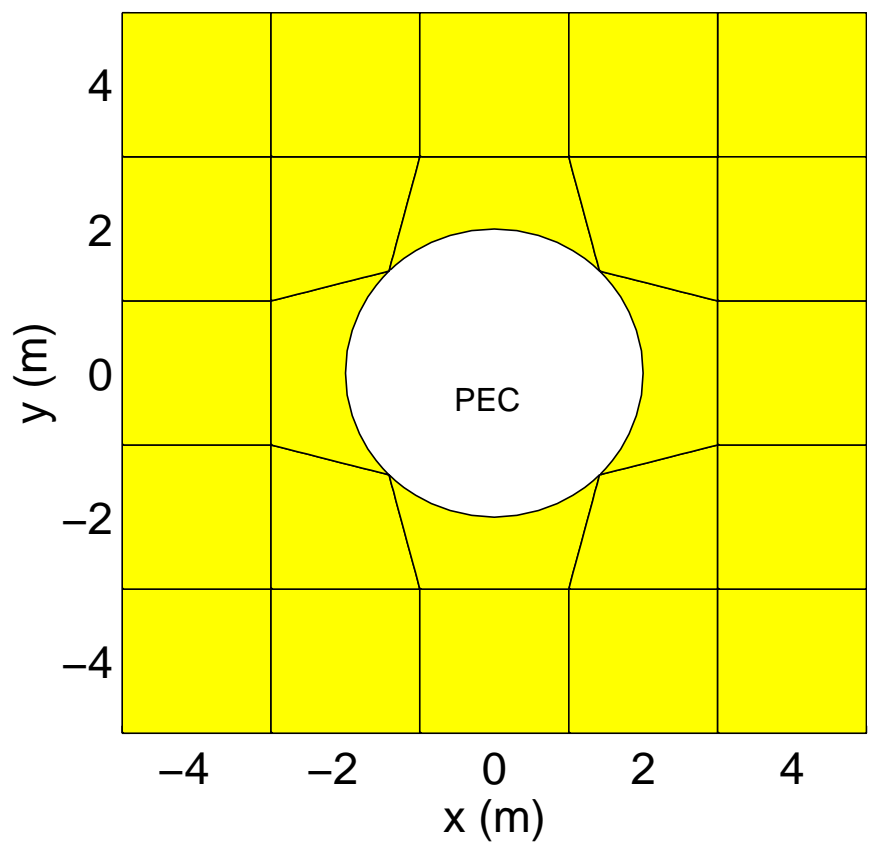

Fig. 3(a)

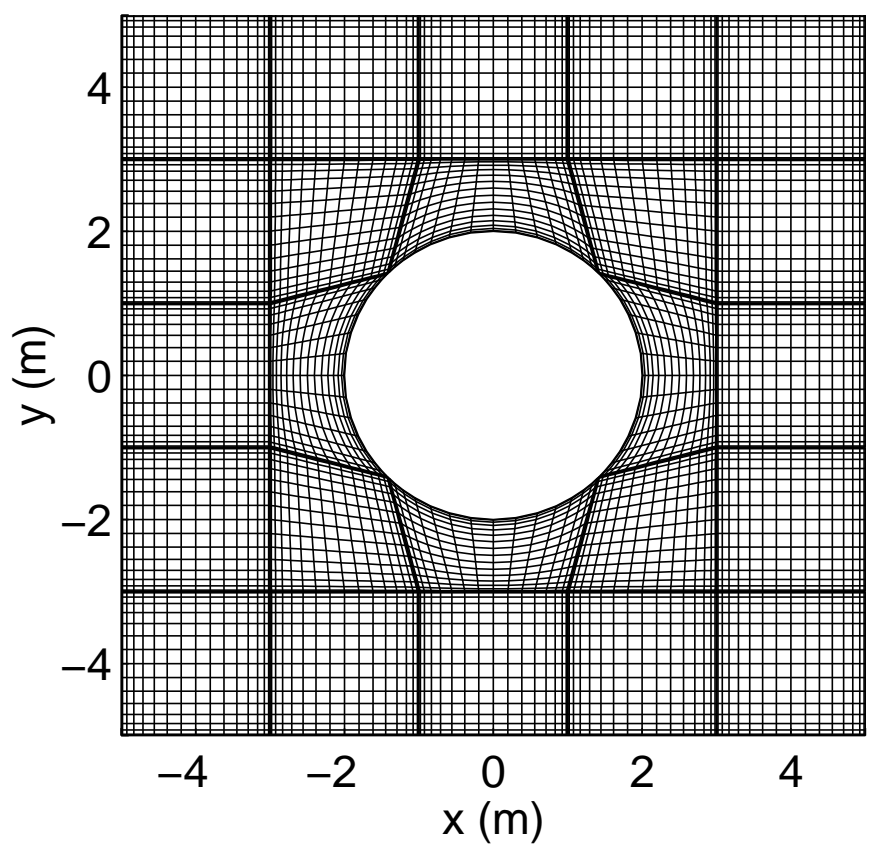

Fig. 3(b) 


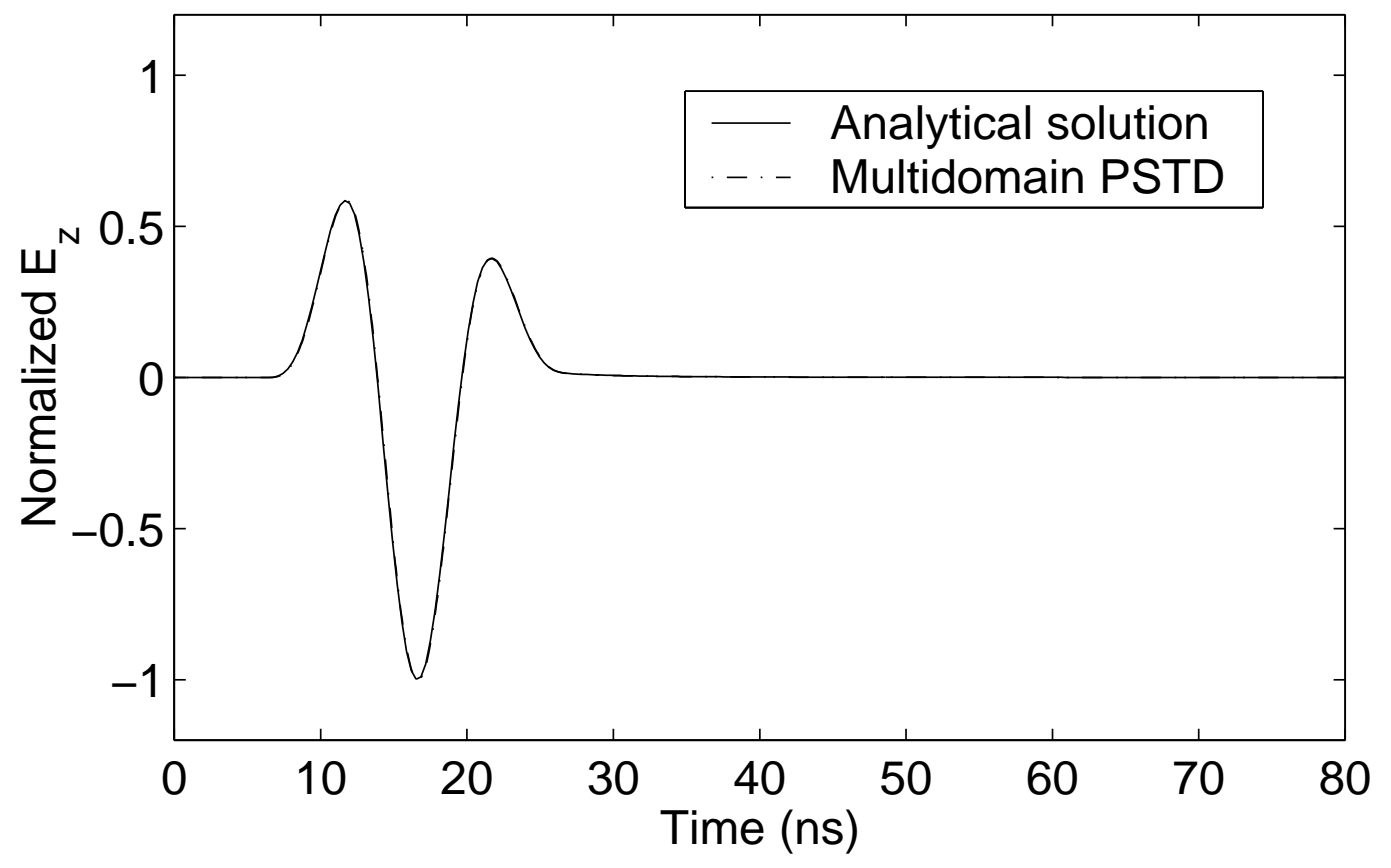

Fig. 3(c) 


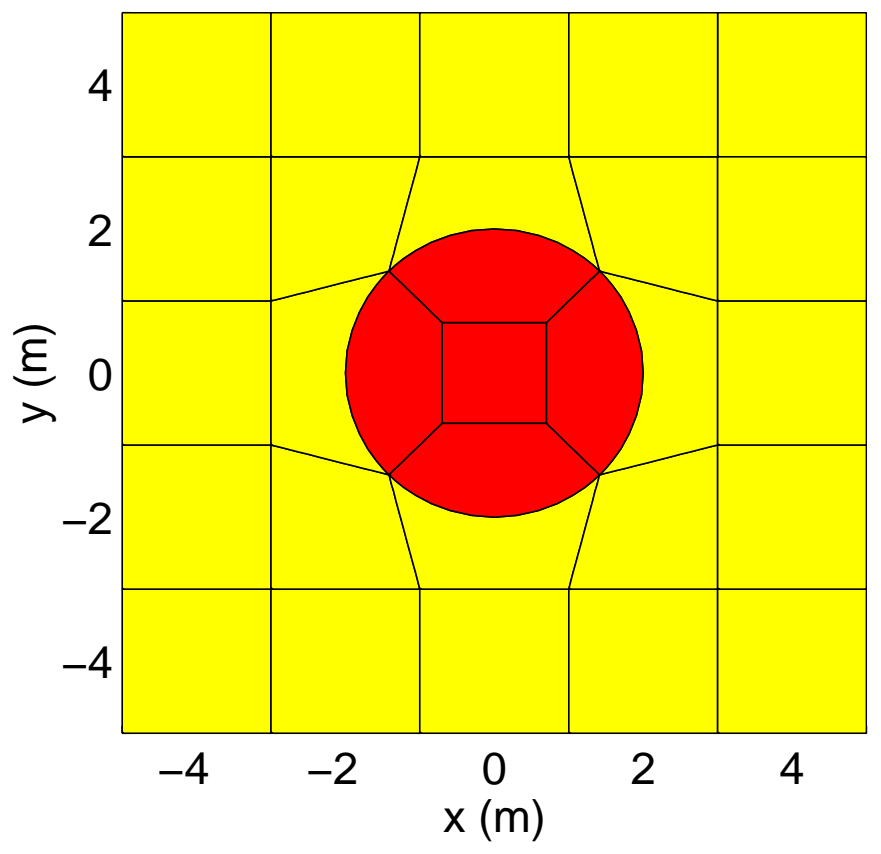

Fig. 4(a)

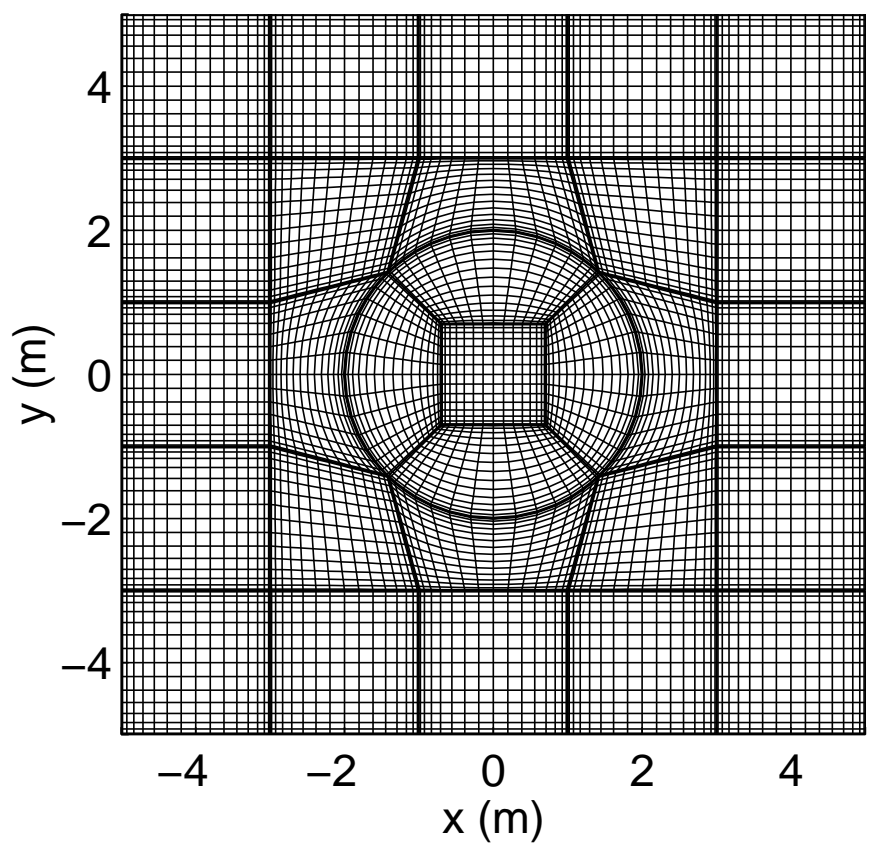

Fig. 4(b) 


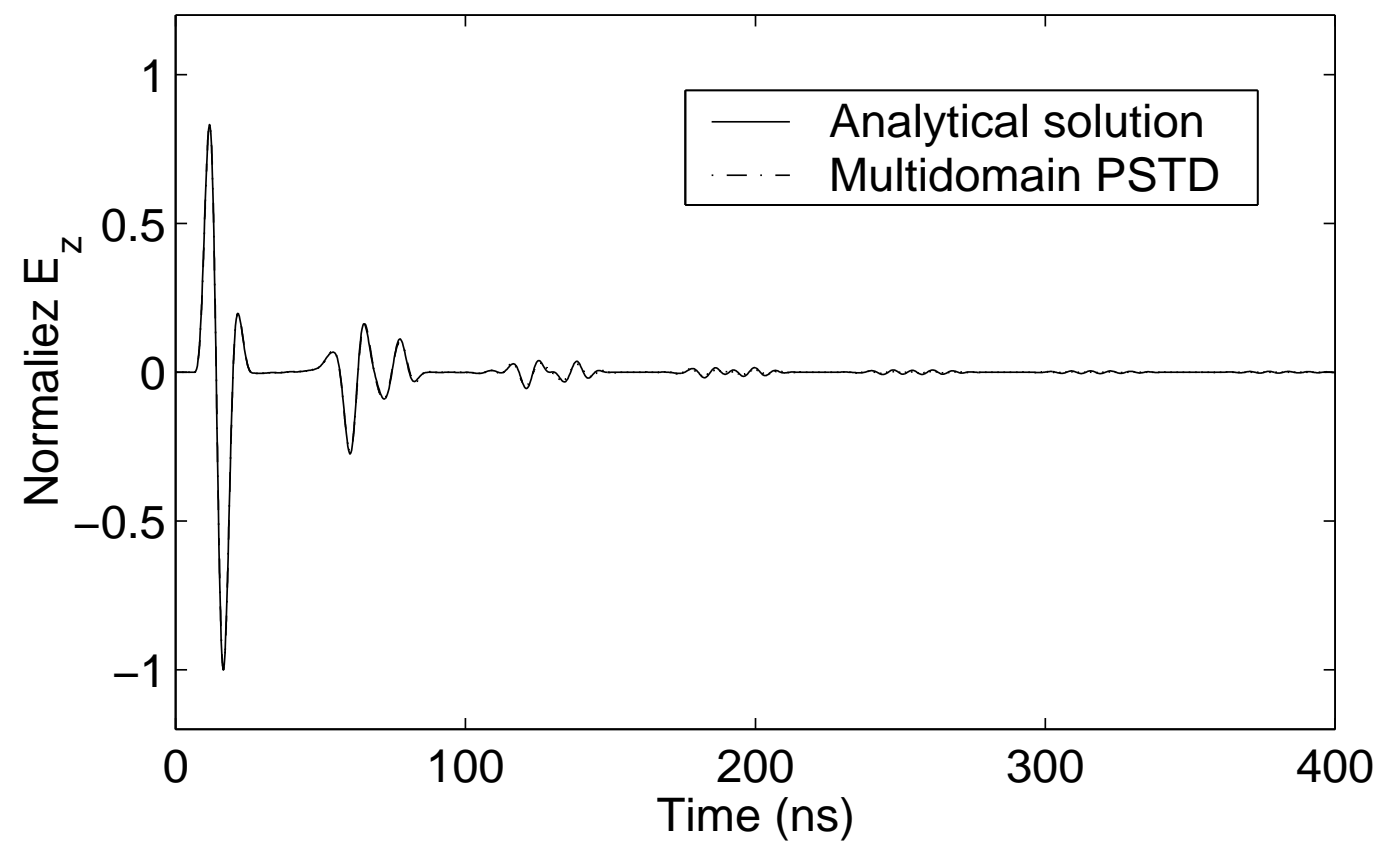

Fig. 4(c) 


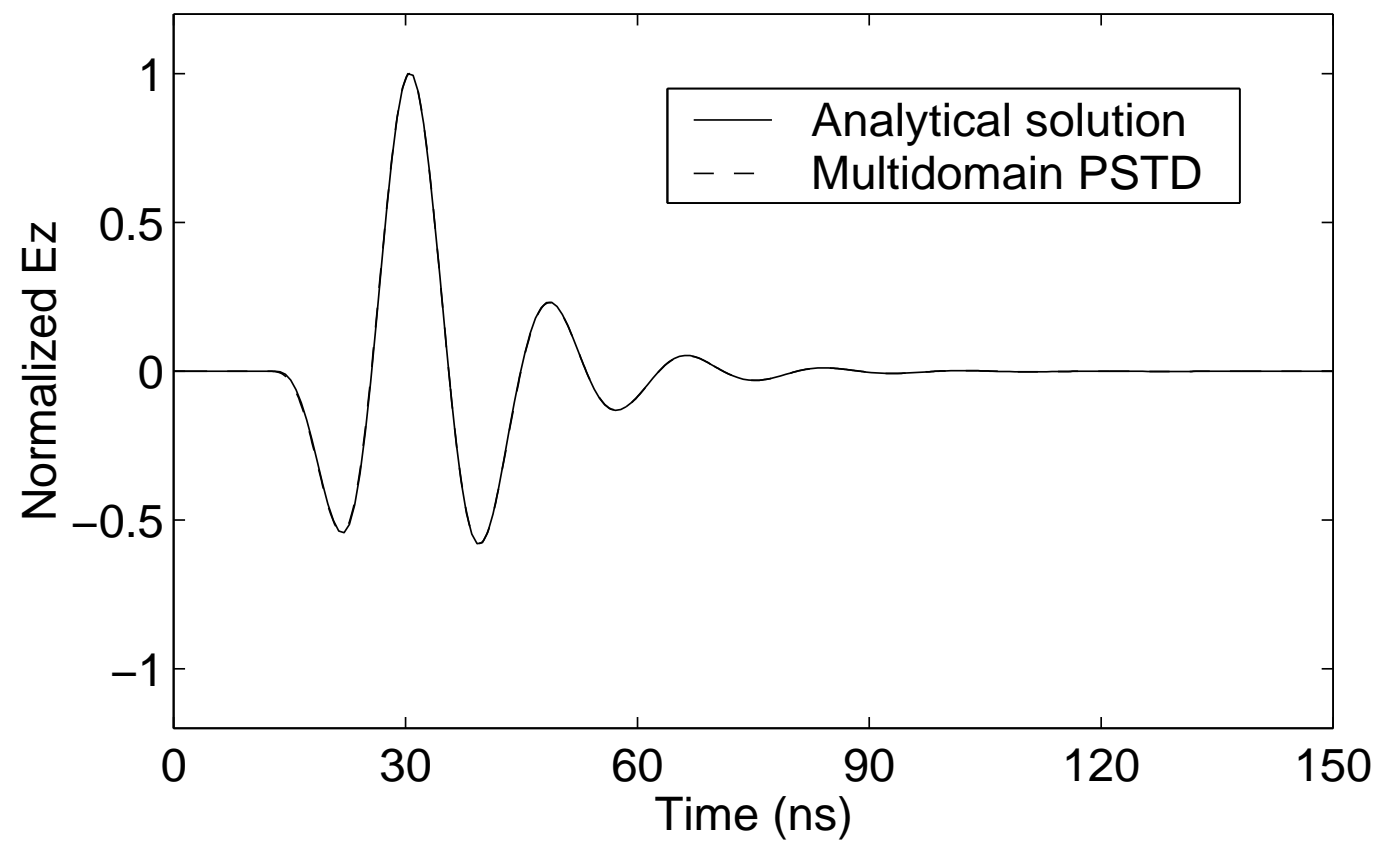

Fig. 5(a)

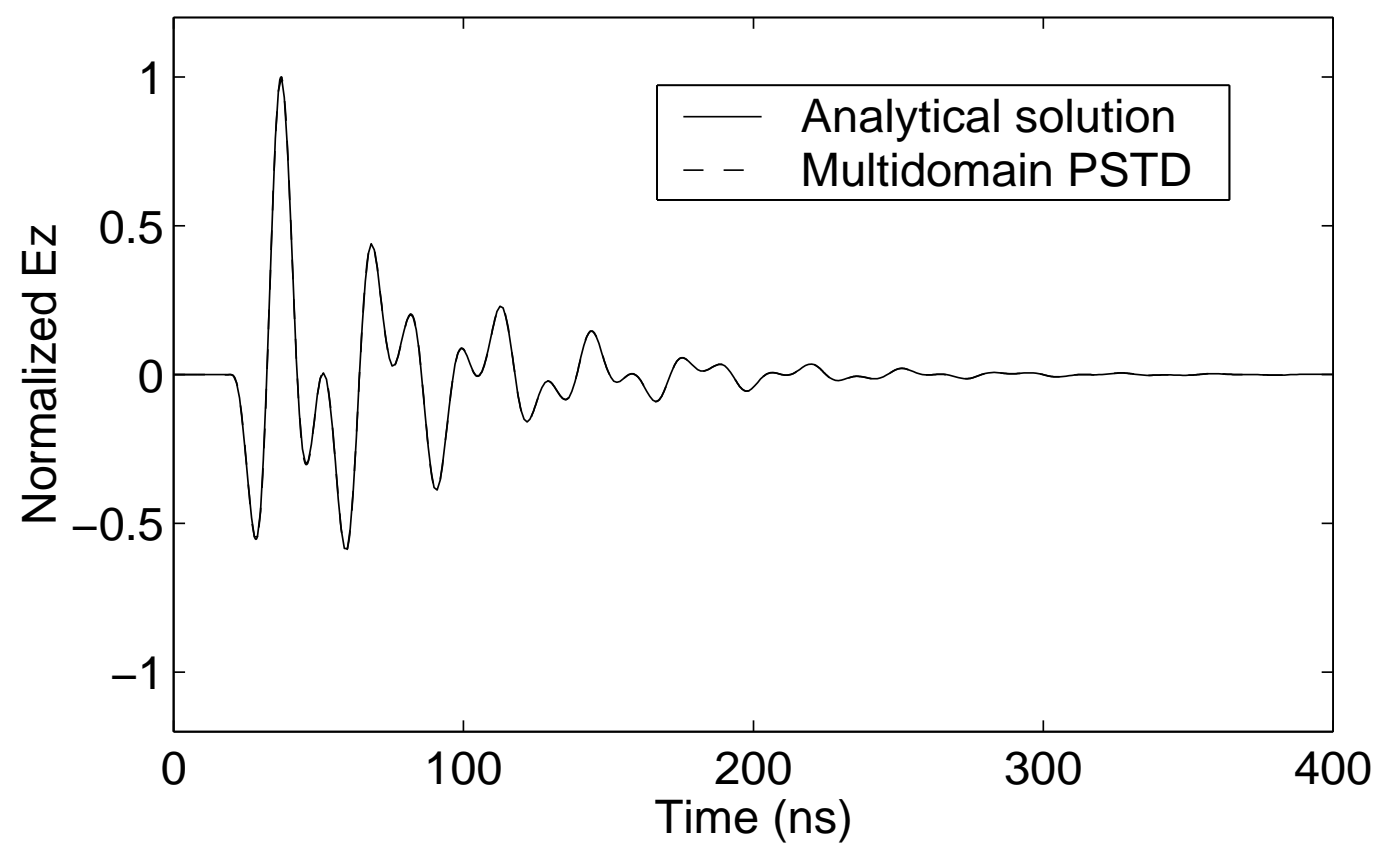

Fig. 5(b) 


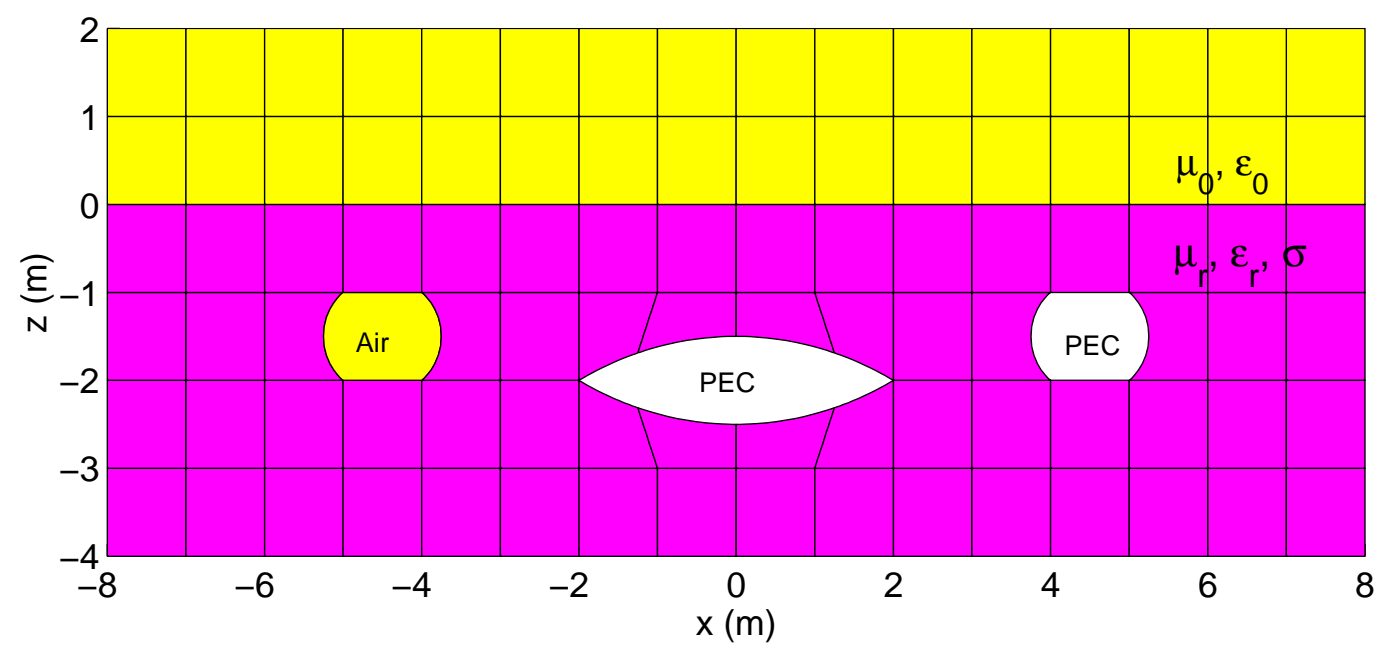

Fig. 6(a)

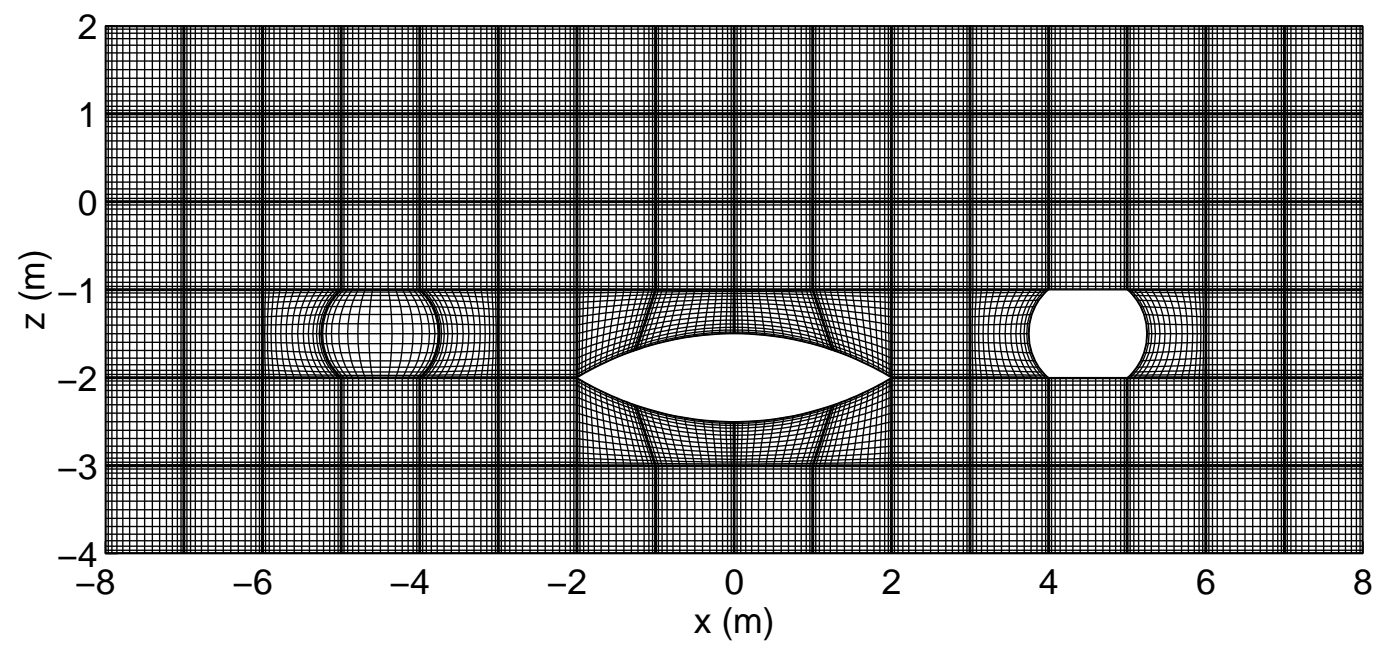

Fig. 6(b) 


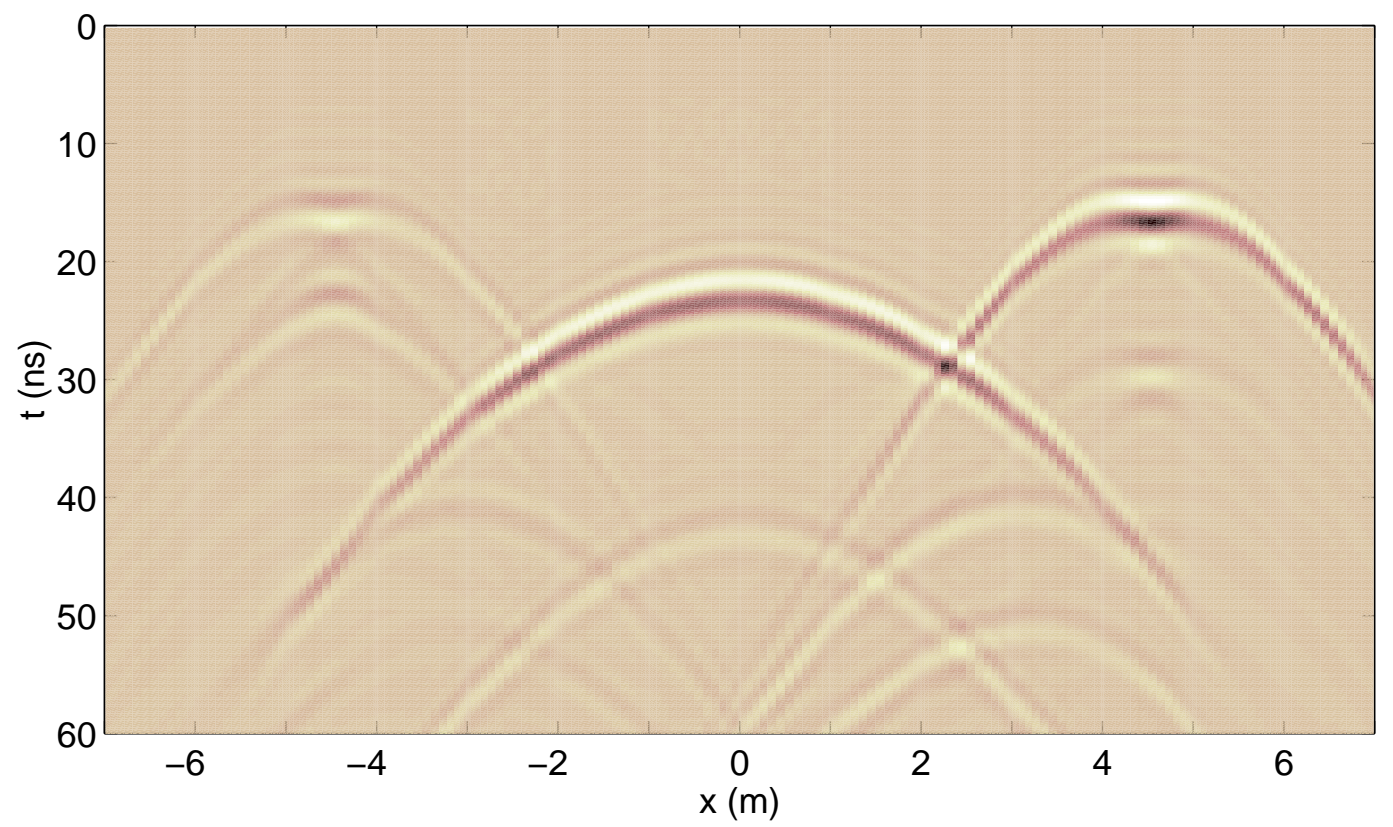

Fig. 6(c) 


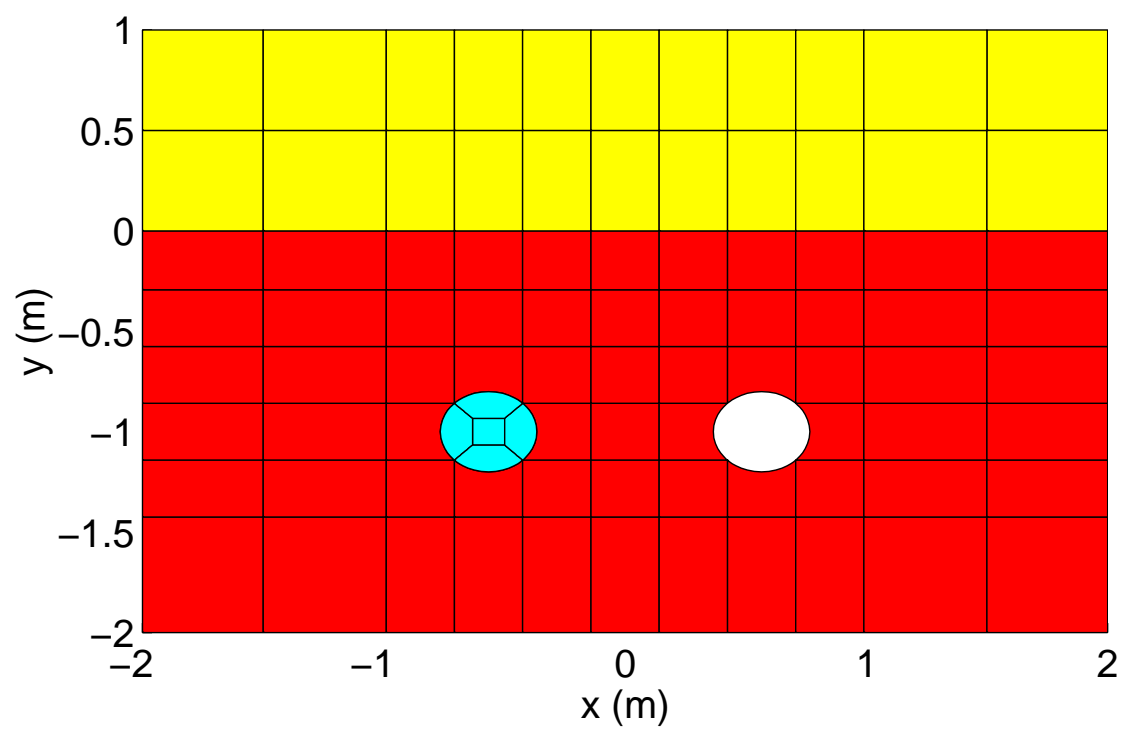

Fig. 7(a)

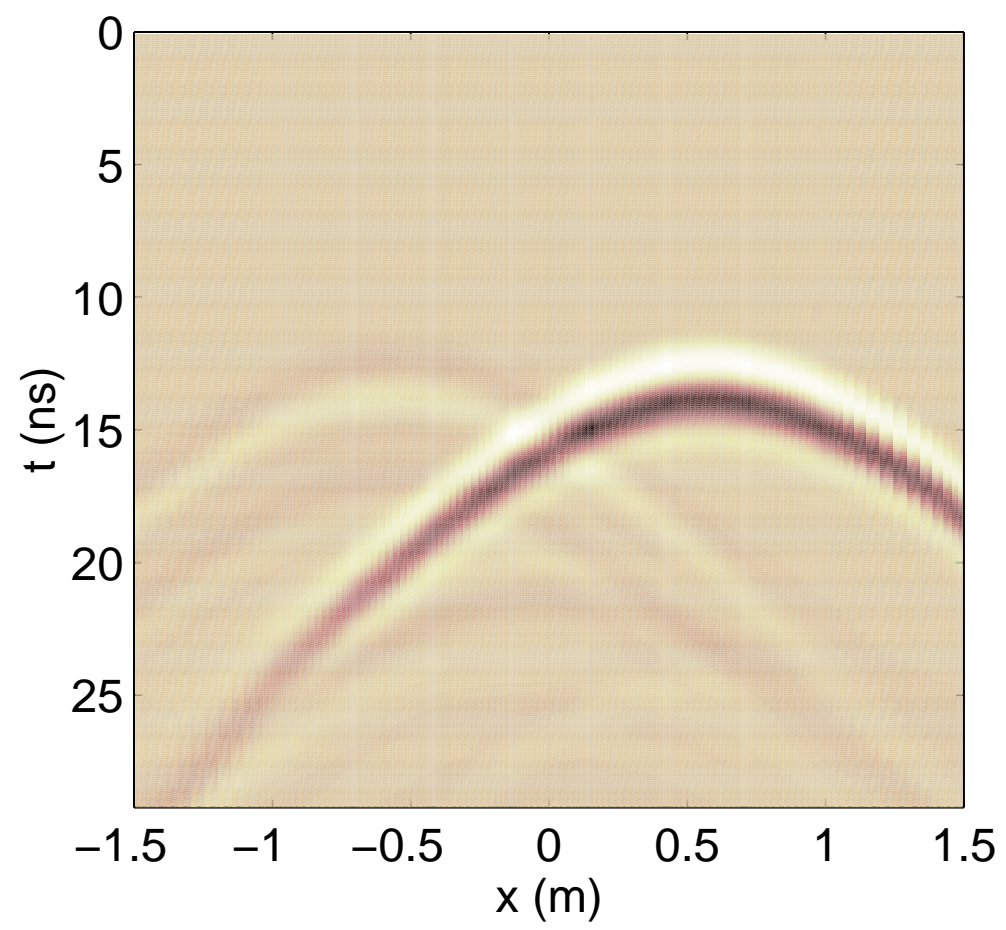

Fig. 7(b) 


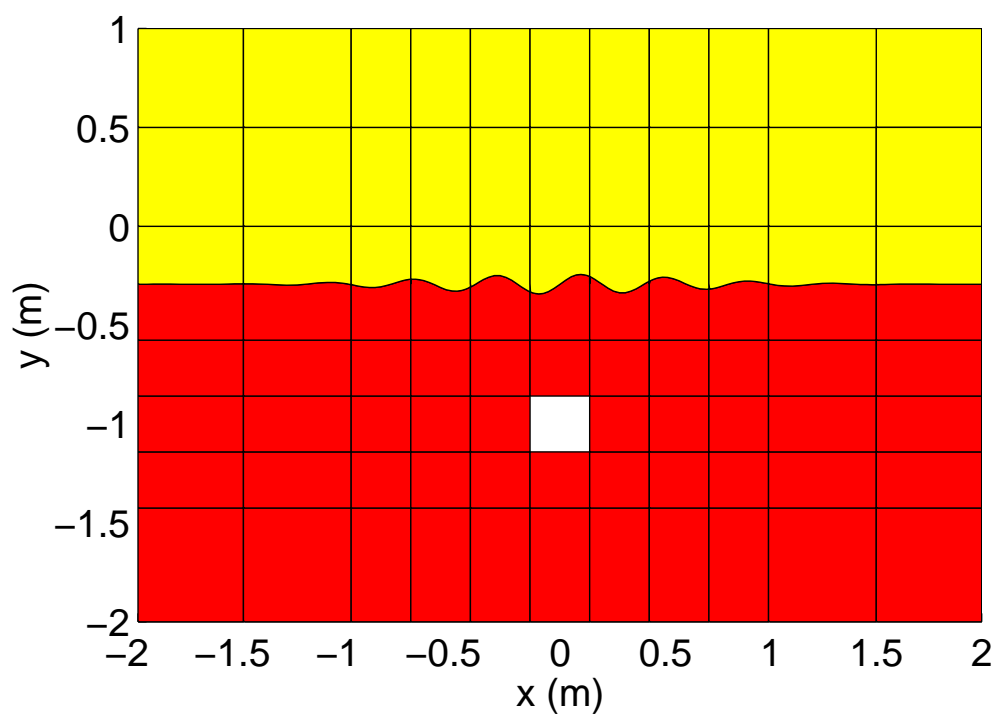

Fig. 8(a)

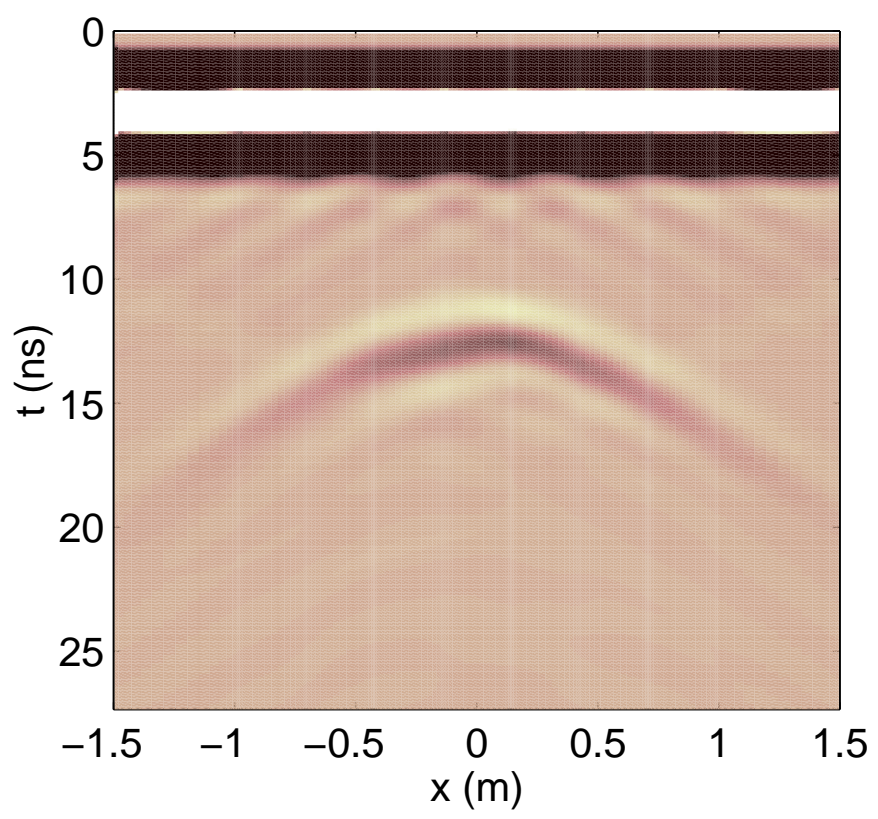

Fig. 8(b) 


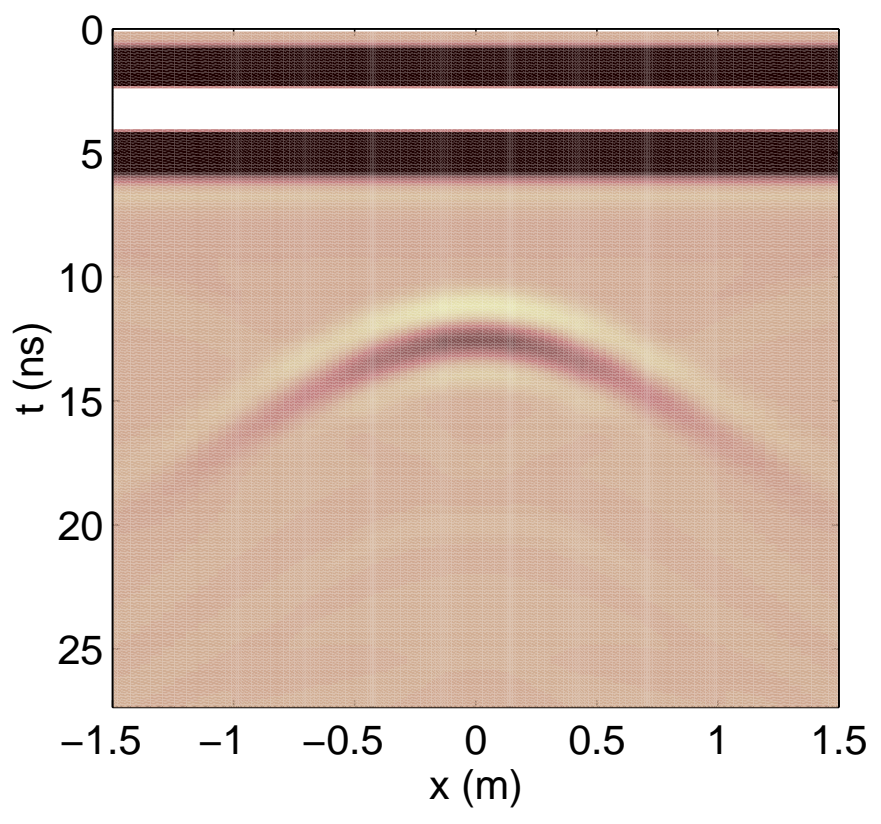

Fig. 8(c) 